\title{
Vitex negundo (Banna) Leaves as Herbal Finish for Cotton Fabric
}

\author{
Sapna Gautam ${ }^{1 *}$, Rajesh Chahota ${ }^{2}$ and Archana Sharma ${ }^{1}$ \\ ${ }^{1}$ Department of Textiles and Apparel Designing, College of Community Science, ${ }^{2}$ Department \\ of Veterinary Microbiology, College of Veterinary \& Animal Sciences, CSK Himachal \\ Pradesh Krishi Vishvavidyalaya, Palampur - 176 062, India \\ *Corresponding author
}

\section{Keywords}

Vitex negundo, Aqueous, Ethanol, Cotton fabric, TPC, Antibacterial

Article Info

Accepted:

10 July 2020

Available Online:

10 August 2020

\begin{abstract}
A B S T R A C T
Herbal extract application on textile substances are in great demand around the globe. It will give a new direction towards the treatment of various diseases through textile industry. Vitex negundo or nirgundi (Banna) is a medicinal plants having antimicrobial properties. Vitex negundo, a large aromatic shrub with typical five foliolate leave pattern, is found throughout the greater part of India at warmer zones and ascending to an altitude of $1500 \mathrm{~m}$ in outer, Western Himalayas. In present study Vitex negundo leave's extract was used to assess the antibacterial activity on cotton fabric. Extraction of phytochemicals was carried out using different solvents like ethanol and aqueous. Total phenolic content (TPC) results revealed that TPC of Vitex negundo leave's extract were highest in case of ethanolic extraction as compared to aqueous. It was observed that mild to moderate antibacterial properties were present in ethanolic extract as compared to aqueous extract. Clear zone of inhibition was observed on cotton fabric against $E$ coli and $S$. aureus. This study thus explored the antimicrobial functionality of cotton fabric coated with plant extract as value added textile product.
\end{abstract}

\section{Introduction}

Textiles are indispensable part of human life. They are mainly to cover the human body for protection against all the adversities. Natural textile fibres are more susceptible to attack than synthetic fibres. Human skin also supports growth of bacteria, due to metabolic side products such as acidic and basic perspiration etc. awareness about eco friendliness in textiles is one of the important issue in recent times since textiles are used next to skin (K. Christie et. al. 2016). Textiles, by virtue of their characteristics and proximity to human body provide an excellent medium for the adherence, transfer and propagation of infection - causing microbial species. When fabric is subjected to laundering, the microbes' gets physically remove from the fabric but is not inactivated. The presence and growth of these microorganism can cause health problems, odour and finally fabric deterioration. Antimicrobial finishes add value to textiles by providing protection through preventing the growth of microorganisms, protect the wearer against microorganisms and protect the textiles itself against bio deterioration. 
An ideal antimicrobial finish for textiles will provide safety, compatibility and durability. Plants are known to produce a variety of compounds which have evolved as defence compounds against microbs and Vitex negundohas shown promise as an effective bio-control agent (Vishwanathan and basavaraju, 2010). Vitex negundo is considered to be very effective in the treatment of various types of disorders in the ayurvedic, homoeopathic and folklore system of medicine in India. Nirgundi (five leaved Chaste tree in English), which means 'protects from all diseases 'in Sanskrit. Vitex negundo belongs to family verbenaceae.

It thrives in humid places or along water courses in wastelands and mixed open forests (Vishwanathan 2010). It has been claimed to possess many medicinal properties (Vishal R. Tandon 2006). Nirgundi is pungent and bitter in taste and has a warming effect. Its leaves are astringent, vermifuge, anti-inflammatory, insecticidal and pesticidal, anti-bacterial, antifungal and analgesic.

The plant also contains alkaloids, glycosides, flavonoids, reducing sugars, sterols, resin and tannins. Some studies have also been done on antimicrobial activity of Vitex negundo along with some other Indian medicinal plants but these works give little information on antimicrobial property of this plant. Hence, in the present experiment an attempt has been made to evaluate the antibacterial activity of leaf extract (ethanol and aqueous) of Vitex negundoas anti-microbial agents on cotton fabric samples using direct dip dry method.

Effectiveness of the finish was studied against Gram Positive and Gram Negative bacteria (Mohanraj, et al., 2012). Phytochemical screening of the extracts were also carried out to assess the presence of different phytochemicals.

\section{Materials and Methods}

\section{Plant source}

The leaves of Vitex negundo were collected from local area of Palampur region of Himachal Pradesh. Collected leaves were cleaned of extraneous matter; shade dried, powdered using mechanical grinder and then were passed through sieve so that uniform powder size is maintained. Dried powder was then kept in air tight containers for further study (Panda et al., 2009).

\section{Preparation of Extract}

\section{Aqueous}

Ten gram of leaves were dissolved in $100 \mathrm{ml}$ of distilled water and kept for overnight. After incubation for 24 hours, the extract was centrifuged and the amount of extract was measured. The final extract obtained was filtered using Whatman filter paper number $40(125 \mathrm{~mm})$, measured, stored in screw caped labelled sample bottles, refrigerated and used for further analysis.

\section{Ethanolic}

Ten gram of leaves were macerated for $24 \mathrm{~h}$ in 70 per cent v/v ethanol. After that vortex for 30 minutes and filtered through Whatman filter paper no. $40(125 \mathrm{~mm})$. The final extract obtained was filtered using Whatman filter paper no. $40(125 \mathrm{~mm})$, supernatant was measured, stored in screw caped labelled sample bottles, refrigerated and used for analysis. Further the aqueous as well as ethanol extract was used for the application on cotton fabric.

Prepared extracts were dried in vacuum oven and powder form of the extract(aqueous and ethnolic)was collected firmly and used for antibacterial study. 


\section{Analysis}

Qualitative phyto-chemical analysis of plant extracts was performed for the identification of various classes of active chemical constituents like alkaloids, flavonoids, phenolic compounds, tannins, saponins and terpenoids using different methods.

\section{Sample preparation for antibacterial study}

Ten percent aqueous as well as ethanol extract was prepared and evaporated in vacuum oven up to $40 \pm 2^{\circ} \mathrm{C}$. After complete evaporation, dried powder was scratched and stored in 20 $\mathrm{ml}$ air tight glass bottles. To study the antibacterial properties of Vitex negundo leaves against $E$ coli and $S$. aureus,10per centextract was prepared using DMSO (Dimethyl sulpho-oxide)solution and then concentration of the extract was increased up to 40 per centto study the clear inhibition zone.

\section{Bioassay of plant extracts}

Bioassay was carried out to assess the antibacterial activity of the plant extracts by Well Diffusion Method to ensure the antibacterial activity of the extracts (Barry, 1980). Muller Hinton Broth culture of the test organisms were firmly seeded overnight in test tubes under $37^{\circ} \mathrm{C} \pm 5^{\circ} \mathrm{C}$ incubation temperature.100 $\mu \mathrm{l} E$ coli and $S$. aureus inoculum was poured on the agar plating and spread firmly using L-shaped spreader. Wells of $6 \mathrm{~mm}$ diameter was punched over the agar plates using a sterile borer. The bottoms of the wells were sealed by pouring $50-100 \mu \mathrm{l}$ of plant extract in the well. Now the plates were incubated at $37^{\circ} \mathrm{C}$ for $18-24 \mathrm{~h}$. After the incubation period formation of zones around the wells, confirms the antibacterial activity of the respective extracts. The same procedure was followed for each strain and extract. Each experiment was carried out in triplicates.

\section{Minimum Inhibitory Concentration (MIC)}

A minimum inhibitory concentration is considered the good standard for determining the susceptibility of organisms to antimicrobials and are therefore used to judge the performance of all other methods of susceptibility testing. The MIC is defined as the lowest concentration of an agent that will inhibit the visible growth of an organism after overnight incubation (Tripathi, 2013).

\section{Stock solution}

$100 \mathrm{ml}$ of antimicrobial stock solution of 10 per cent concentration (10 $\mathrm{g}$ of plant source in $100 \mathrm{ml}$ of solvents) was prepared.

\section{Culture}

24 hours subculture of the test organism $S$. aureus (Gram positive) and E. coli (Gram negative) was serially diluted and $1 \times 10^{-5}$ dilution was selected for MIC study.

A separate test tube containing nutrient broth (test tube 1) alone was used as control. All the test tubes and control sample were incubated at $37^{\circ} \mathrm{C}$ for 24 hours. After the period of incubation, MIC was determined on the basis of turbidity that occurred.

\section{Pre-treatment of textile material: Desizing}

\section{Recipe}

Sodium hydroxide $-2 \%$ owf (on weight of fabric)

Detergent $-2 \%$ owf

Material to liquor Ratio - 1:40

Temperature $-40-60{ }^{\circ} \mathrm{C}$

The weighed fabric, amount of sodium hydroxide detergent and material to liquor ratio was calculated accordingly. Added sodium hydroxide and detergent in water and 
heat the solution to $40^{\circ} \mathrm{C}$, then immersed the fabric in the solution and treat for one hour at $40-60^{\circ} \mathrm{C}$, later the fabric was washed thoroughly under running water to remove traces of sodium hydroxide and shade dried (Sumithra and Raaja, 2013).

\section{Finishing}

\section{Direct method}

\section{Recipe}

Material to liquor Ratio - 1:40

Antimicrobial stock solution - concentration as per MIC study (owf) i.e. $10 \%$

Crosslinking agents (citric acid) $-6 \%$

Immersed the pre-treated cotton fabric in the antimicrobial stock solution in aqueous and ethanol extract without cross linking agent and also in stock solution containing 6 per cent cross linking agent i.e. citric acid for one hour and then cured the treated fabrics in oven for $30 \mathrm{sec}$. and then shade dried.

\section{Antimicrobial activity - Parallel streak method (AATCC 147)}

Parallel streak method is a qualitative antimicrobial test used to detect diffusible bacteriostatic activity on textile materials. This method is useful for obtaining a rough estimate of antibacterial activity by the size of the zone of inhibition caused by the presence of the antibacterial agent.

Materials required - Autoclave, laminar air flow chamber, sterile petri plates, incubator, micropipettes, inoculation loop, sterile forceps, test specimens

Media - Nutrient agar and nutrient broth

Test organisms - Staphylococcus aureus (S aureus) and Escherichia coli (E coli)
Sterilized nutrient agar was poured in petri plates and allowed to solidify firmly before inoculating. Prepared inoculum by transferring $1 \pm 0.1 \mathrm{ml}$ of 24 hours old broth culture into $9 \pm 0.1 \mathrm{ml}$ sterile test tubes and mixed properly. Incubated overnight and then one loopful of diluted inoculum poured on petri plate by making five streaks of $60 \mathrm{~mm}$ length spacing $10 \mathrm{~mm}$ apart, covering the central area of the petri plates without refilling the loop.

The streak lines were without any break in inoculation or on the media. Gently pressed the test specimens of size $25 \mathrm{~mm} \times 50 \mathrm{~mm}$ transversely across the five streaks to ensure the intimate contact with the agar surface. The plates were then incubated at $37 \pm 2{ }^{\circ} \mathrm{C}$ for 24 hours. Examined the incubated plates for interruptions of bacterial growth along the streaks of inoculum, beneath the specimen and beyond the fabric edge (Anonymous, 2013).

\section{Performance of fabric treated with herbal finish}

Performance of fabric treated with herbal finish was observed using the parameters like fabric thickness (mm), fabric count (no.) and gram per square meter (GSM).

\section{Results and Discussion}

Extract from dried powder of Vitex negundo (Banna) leaves was prepared using 70\% ethanol and aqueous solution. After extraction quantity of extract obtained was measured and less difference was observed in both extraction methods (Table.2).

There are several types of solvents that can be used for extraction of plant extract such as methanol, water, ethanol, acetone etc. Rabeta and An Nabil 2013 reported the presence of TPC in the leaves of Vitex negundo Linn. 
Total phenolic content (TPC) of Vitex negundo were analysed as high (113.88 mg / g GAE) in ethanol extract and low in (72.10 $\mathrm{mg} / \mathrm{g}$ GAE) aqueous extract.

During qualitative analysis of phyto-chemical in Vitex negundo Leaves (Table.2) using aqueous and ethanol mediums, it was observed that alkaloids, flavonoids, phenolic compounds, tannins, saponin and terpenoid compounds were present in Vitex negundo aqueous as well as ethanolic leave's extract.

Antibacterial activities in selected plant sources in Table 3 exhibits that Vitex negundo extraction in 70 per cent ethanol showed moderate sensitive properties against $E$ coli (Gram negative) as compared to mild sensitive properties against $S$. aureus.

Table.1 Preparation of tubes for MIC

\begin{tabular}{|c|c|c|c|c|c|c|c|c|c|c|c|}
\hline Test tubes & 1 & 2 & 3 & 4 & 5 & 6 & 7 & 8 & 9 & 10 & 11 \\
\hline Volume of nutrient broth (ml) & 10 & 9 & 8 & 7 & 6 & 5 & 4 & 3 & 2 & 1 & 0 \\
\hline Volume (V2) antimicrobial stock solution (ml) & 0 & 1 & 2 & 3 & 4 & 5 & 6 & 7 & 8 & 9 & 10 \\
\hline Concentration (\%) & 0 & 1 & 2 & 3 & 4 & 5 & 6 & 7 & 8 & 9 & 10 \\
\hline Bacterial culture $(\mathrm{ml})\left(1 \times 10^{-5}\right.$ Conc. $)$ & 0.1 & 0.1 & 0.1 & 0.1 & 0.1 & 0.1 & 0.1 & 0.1 & 0.1 & 0.1 & 0.1 \\
\hline
\end{tabular}

Table.2 Yield of extracts

\begin{tabular}{|l|l|c|c|}
\hline Plant Source & Part used & \multicolumn{2}{|c|}{ Yield of extracts (ml/50ml) } \\
\cline { 3 - 4 } & & Ethanol(70\%) & Distilled water \\
\hline Vitex negundo & Leaves & 32 & 26 \\
\hline
\end{tabular}

Table.3 Phyto-chemical analysis of Vitex negundo leaves extract

\begin{tabular}{|l|l|c|c|}
\hline S.No & Phyto-chemical tests & Aqueous solution & Ethanol \\
\hline $\mathbf{1}$ & Test for Alkaloids & & \\
\hline & Dragendorff's reagent & - & + \\
\hline & Wagner's reagent & + & + \\
\hline $\mathbf{2}$ & Test for Flavonoids & & \\
\hline & Ammonia test & + & - \\
\hline & Sodium Hydroxide test & + & + \\
\hline $\mathbf{3}$ & Test for phenolic compounds and tannins \\
\hline & Ferric chloride reagent & + & + \\
\hline & Gelatin reagent & - & + \\
\hline & Lead acetate reagent & - & - \\
\hline $\mathbf{4}$ & Test for saponins & & \\
\hline & Foam test & + & + \\
\hline $\mathbf{5}$ & Test for terpenoids & & \\
\hline & Salkowski test & + & + \\
\hline
\end{tabular}


Table.4 Antimicrobial activity of Vitex negundo leaves extract

\begin{tabular}{|l|l|l|l|}
\hline \multicolumn{4}{|c|}{ Antimicrobial activity } \\
\hline \multicolumn{3}{|c|}{ S. aureus } & E. coli \\
\hline 70\% ethanol & Distilled water & 70\% ethanol & Distilled water \\
\hline MS I+ & - & MS II+ & MS I+ \\
\hline
\end{tabular}

Table.5 Minimum Inhibitory Concentration (MIC) of Vitex negundo

\begin{tabular}{|c|c|c|c|c|c|c|c|c|c|c|}
\hline \multirow[t]{2}{*}{ Herbal extract } & \multicolumn{10}{|c|}{ Minimum Inhibitory Conc. (Turbidity/ no turbidity) } \\
\hline & 1 & 3 & 4 & 5 & 6 & 7 & 8 & 9 & 10 & 11 \\
\hline Banna (Vitex negundo) & Turbidity & & & & & & & & & \\
\hline
\end{tabular}

Table.6 Performance of cotton fabric treated with Vitex negundo as herbal finish

\begin{tabular}{|l|l|l|l|l|l|}
\hline $\begin{array}{l}\text { Cross linking } \\
\text { agents }\end{array}$ & \multicolumn{4}{|c|}{ Parameters } & \\
\hline & Thickness (mm) & $\begin{array}{l}|c| \\
\text { Direct method }\end{array}$ & \\
\hline $\begin{array}{l}\text { Count (no.) } \\
\text { (without) }\end{array}$ & 0.31 & GSM & Strength (kgf) & Elongation (\%) \\
\hline Citric acid & 0.72 & 1.467 & 23.1 & 13.0 \\
\hline $\begin{array}{l}\text { 82 picks / inch } \\
100 \text { ends / inch } \\
78 \text { picks / inch }\end{array}$ & 2.198 & 24.8 & 9.0 \\
\hline
\end{tabular}

Table.7 Antibacterial activity of herbal treated fabrics

\begin{tabular}{|l|l|l|}
\hline Cross linking agents & S. aureus & E. Coli \\
\hline Control (without) & - & - \\
\hline Citric acid & MS I+ & MS II+ \\
\hline
\end{tabular}

Fig.1

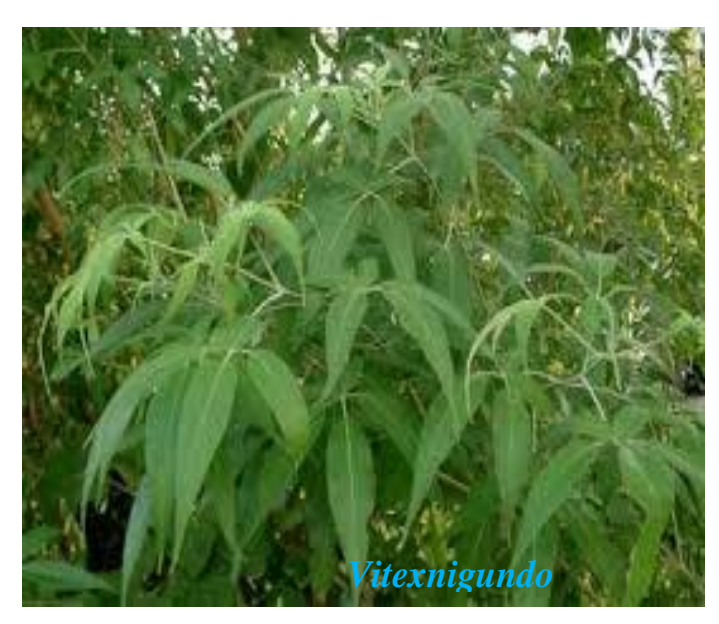


Fig.2

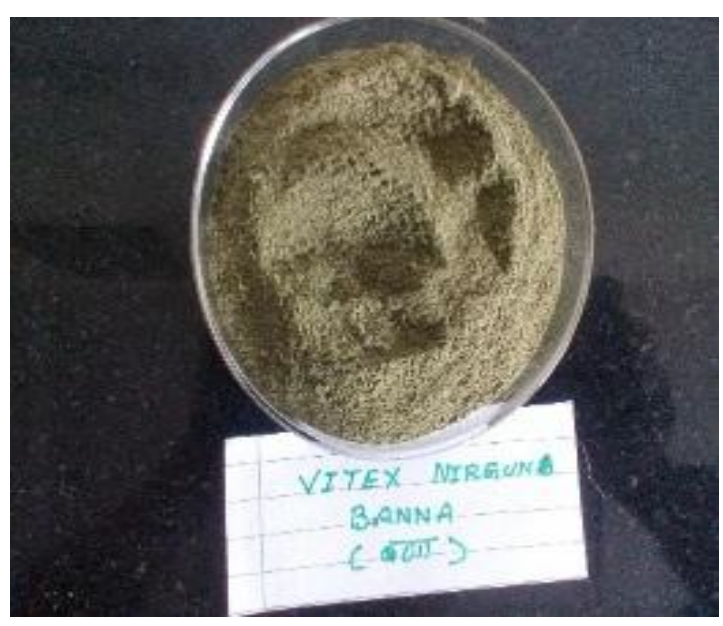

Fig.3

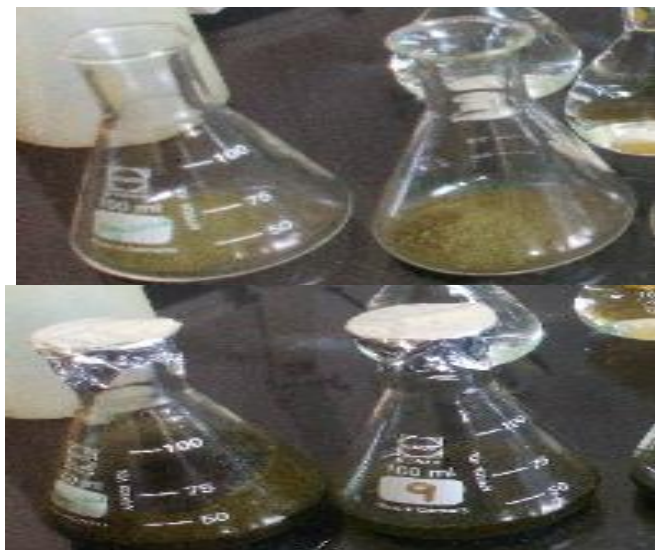

Fig.4

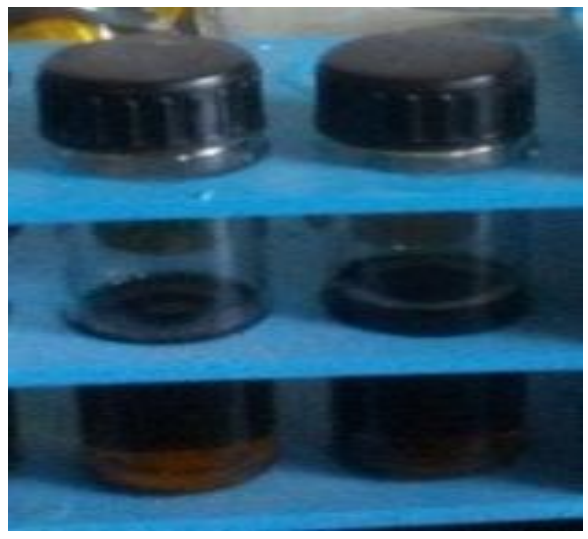


Fig.5 Total phenolic content (TPC) of plant extracts

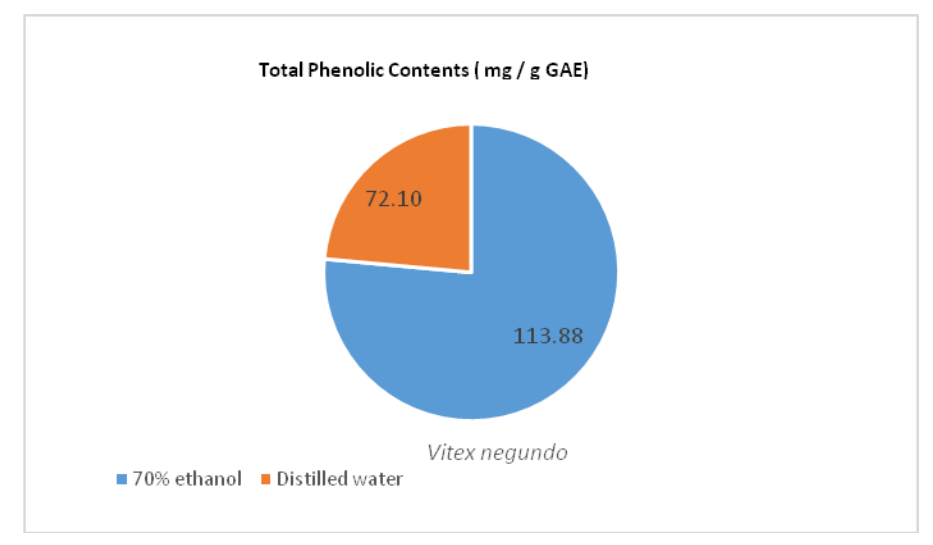

Fig.6
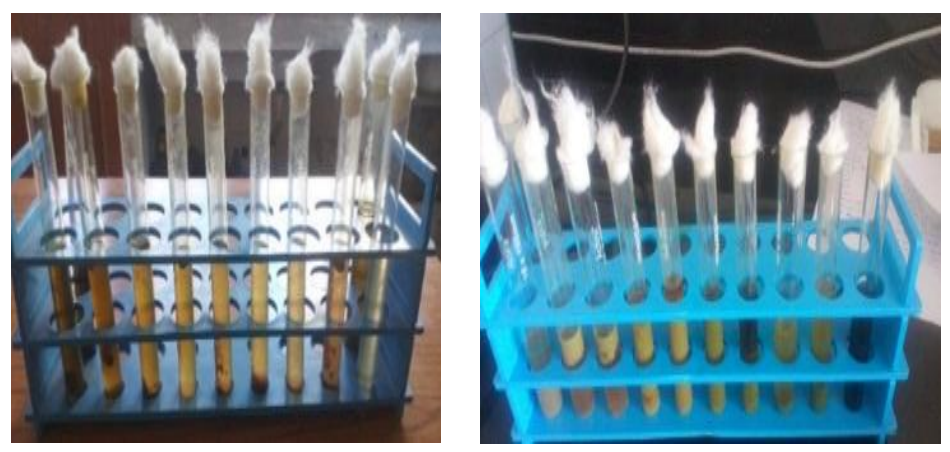

Fig.7
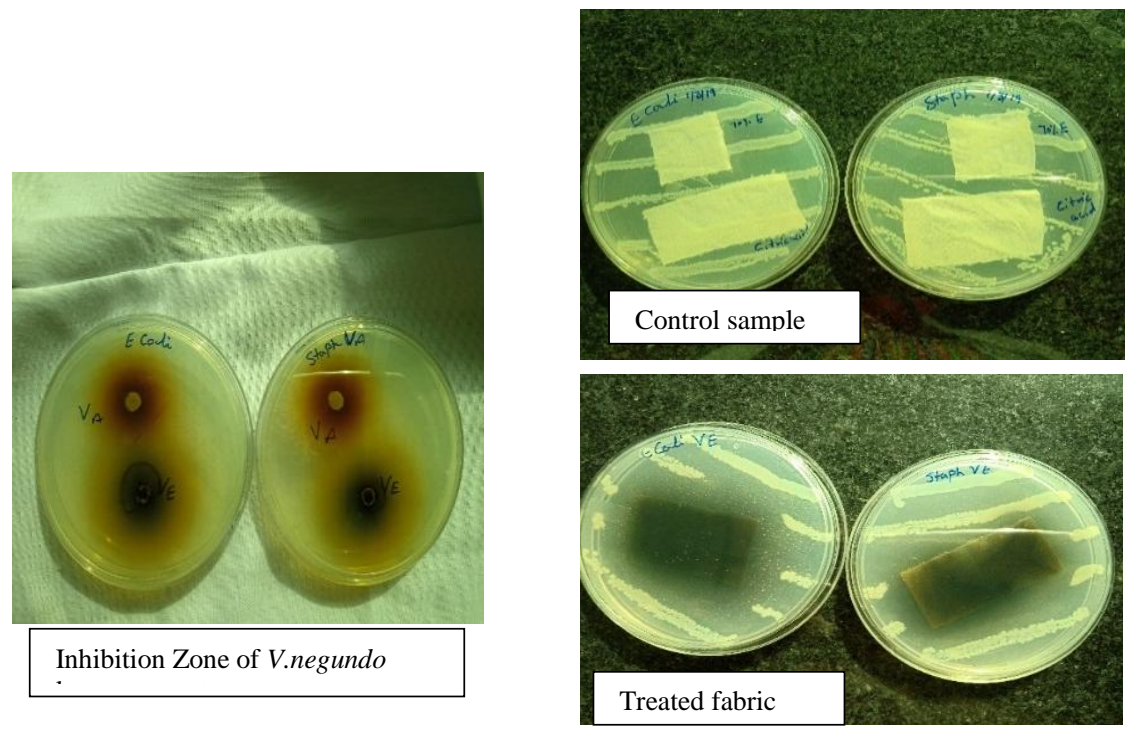
MS I+ - Mild sensitive MS II+ - Moderate sensitive

Minimum Inhibitory Concentration of selected herbal extract was determined to study the susceptibility of selected herbal finishes against $S$. aureus organisms. As the extracts used were coloured in nature so when $S$. aureus and $E$ coli cultures were inoculated in the dilution $1 \times 10^{-5}$, turbidity was observed so the 10 per cent concentration of herbal extract was used during application on cotton fabric.

Thickness of fabric treated using herbal finish from Vitex negundo with citric acid as crosslinking agent was observed as higher i.e. $0.72 \mathrm{~mm}$ as compared to control fabric sample. Slight increase in GSM was also observed as 1.467 and 2.198 in control and treated samples respectively. Strength of cotton fabric treated with selected herbal finish was analysed as 23.1 to $24.8 \mathrm{kgf}$ in control and cross linked samples respectively whereas elongation was calculated as 13.0 per cent in control samples to 9.0 per cent in treated samples respectively.

Antibacterial activity of Vitex negundo treated fabrics was observed against $S$. aureus (Gram positive) and E. coli (Gram negative) bacteria and clear zone of inhibition was observed. Same results were reported by $S$ Mohanrajet.al 2012, when leaf extracts of Vitex negundo are applied directly and in encapsulated form on to the fabric samples.

Potentially, the extract of Vitex negundo can be used for bactericidal applications on textile materials. Plant based antimicrobial agents have therapeutic potential as they do not impose any side effects to the human beings. This study is particularly significant in the environment containing both types of organisms for coating cloths with eco-friendly natural plant extract. Antimicrobial agents derived from natural sources are safe for human and the environment.

\section{References}

Anonymous, 2013. Training programme for testing antimicrobial textiles, The synthetic \& art Silk Mills' Research Association (Sasmira), Mumbai, Pp 3744.

Barry, A., L, 1980. Procedure for testing antimicrobial Agents in Agar media. In: Antibiotics in Laboratory Medicine. Lorin V (eds), Williams Wilkins Co. Baltimore: USA, pp. 1-23.

Christie Jennifer, K., R. Pragadheeswari and K. Sangeetha, 2016. Herbal finishes on cotton fabric using Acorus calamus (Vasambu) extract. International Journal of Science Technology and Management, ISSN 2394-1537.

Mohanraj, S, P Vanathi, N Sowbarniga and D Saravanan, 2012. Antimicrobial effectiveness of Vitex negundo leaf extract. Indian Journal of Fibre and Textile Research, pp 389-392.

Panda, S. K., H. N. Thatoi and S. K. Dutta, 2009.Antibacterial activity and phytochemical screening of leaf and bark extracts of Vitex negundo from similipal biosphere reserve, Orissa. Journal of Medicinal Plants Research, 3(4), pp 294-300.

Rabeta, M.S. and An Nabil, Z., 2013. Total phenolic compounds and scavenging activity in Clitoria ternatea and Vitex negundo Linn. International food Resaerch Journal, 20(1), 495-55.

Sumithra, M and Raaja, V.N., 2013. Effect of medicinal herb extracts treated on cotton denim fabric. Mintage J. Pharmaceut. Med. Sci., 2 (4): 6-9.

Tripathi, K.D., 2013. Essentials of Medical Pharmacology $\left(7^{\text {th }}\right.$ ed.). New Delhi, India: Jaypee Brothers Medical Publishers, Pp 696-697. 
Vishal R. Tandon \& R.K. Gupta. 2006.Vitex negundo Linn (VN) leaf extract as an adjuvant therapy to standard antiinflammatory drugs. Indian J Medical Research, 124. pp 447-450.
Vishwanathan, S. and R. Basavaraju. 2010.A Review on Vitex negundo L. - A Medicinally Important Plant. European Journal of Biological Sciences,3 (1):3042.

\section{How to cite this article:}

Sapna Gautam, Rajesh Chahota and Archana Sharma. 2020. Vitex negundo (Banna) Leaves as Herbal Finish for Cotton Fabric. Int.J.Curr.Microbiol.App.Sci. 9(08): 379-388. doi: https://doi.org/10.20546/ijcmas.2020.908.044 